\title{
ON AFFINE SYMMETRIC SPACES
}

\author{
BY \\ SEBASTIAN S. KOH
}

1. Introduction. An affine symmetric space is a connected affinely connected manifold $M$ such that to each point $p \in M$ there is an involutive (i.e., of order 2) affine transformation $s_{p}$ of $M$ leaving $p$ as an isolated fixed point. Clearly, for a fixed $p$ in $M$, the transformation $s_{p}$ is unique; it is called the symmetry at $p$.

It is well known that the group of affine transformations $A(M)$ of $M$ is a Lie group and that, if $M$ is affine symmetric, the connected component of identity $A_{0}(M)$ of $A(M)$ acts transitively on $M$ (see e.g. [4]). Let $G=A_{0}(M)$ and let $H$ be the isotropy subgroup of $G$ at $p \in M$. Then $M$ can be identified with $G / H$, the left coset space, and $s_{p}$ induces an involutive automorphism $\Sigma: g \rightarrow s_{p} \circ g \circ s_{p}$ such that $G_{0}^{\Sigma} \subset H \subset G^{\Sigma}$, where $G^{\Sigma}$ denotes the subgroup of fixed points of $\Sigma$ and $G_{0}^{\Sigma}$ is the connected component of identity in $G^{\mathbf{\Sigma}}$.

Conversely, if $G$ is a Lie group with an involutive automorphism $\Sigma$ and $H$ is a subgroup satisfying $G_{0}^{\Sigma} \subset H \subset G^{\Sigma}$, then $H$ is a closed subgroup of $G$ and the coset space $G / H$ is an analytic manifold; furthermore, the canonical invariant affine connection of $G$ induces an affine connection on $M=G / H$, which renders $M$ an affine symmetric space with symmetries derived from $\Sigma$ in an obvious manner. Such an affine symmetric space will be denoted by $(G / H, \Sigma)$ or simply by $G / H$. The discussion given in the preceding paragraph shows that we may restrict our study of affine symmetric space to the case $M=G / H$, where $G$ is a connected Lie group. We may, and we shall, further assume that $G / H$ is effective in the sense that $H$ contains no nontrivial normal subgroup of $G$; for if we take $G=A_{0}(M)$, then any normal subgroup $H^{\prime} \subset H$ induces identity transformation on $M$.

In general, if $G$ is a group of affine transformation of a connected, affinely connected manifold $M$, then the isotropy subgroup $H_{p}$ of $G$ at $p \in M$ is isomorphic to the linear isotropy subgroup $\tilde{H}_{p} \subset G L\left(M_{p}\right)$, where $M_{p}$ denotes the tangent space to $M$ at $p$. It follows that the affine symmetric space $(G / H, \Sigma)$ is a riemannian symmetric space if, and only if, $H$ is compact. The riemannian case has been extensively studied since É. Cartan inaugurated this subject some thirty-five years ago. The general case was first systematically studied by K. Nomizu in 1954, and then by $M$. Berger. One remarkable theorem, relating these two cases, is given by Berger in [1]; it asserts that every affine symmetric space is fibered over a riemannian one with fibers homeomorphic to a euclidean space. However the

Received by the editors June 18, 1964. 
proof of Berger, based on a theorem of G. D. Mostow on covariant fibering (see [5]), is incomplete. Our Theorem 1 sharpens Berger's result in that our fibration respects the symmetries in the symmetric space.

This paper divides naturally into two parts. The first part consists of $\S \S 2$ and 3 , and deals with the topological structure of affine symmetric spaces. Our main result is Theorem 1 mentioned above, and our tool is the Main Lemma stated in $\S 1$, which is proved by using a technique developed by Mostow in [5]. The corollary to the Main Lemma is of some interest in itself.

Using Lemma A, we see that an easy proof of the following theorem of Chevalley can be extracted from Mostow's proof of Theorem 2.1 in [5]: Let $G$ be a connected solvable Lie group and let $C$ be any closed connected subgroup of $G$. Then the homogeneous space $G / C$ is homeomorphic to a product of circles and lines.

The second part is devoted to the local study of affine symmetric spaces or, equivalently, the study of involutive Lie algebras. The simple involutive Lie algebras have been classified by Berger. The method used by him consists of a list of case by case studies and gives little insight to the structure of such algebras. We have completely determined the local structure of such affine symmetric spaces $G / H$ where $G$ is semi-simple and $H$ is not semi-simple. They include the pseudo-hermitian symmetric spaces. In particular, we have a simple and elementary proof of the fact that the complex structure $J$ of a pseudo-hermitian symmetric space is induced by an element in the center of the Lie algebra of the linear isotropy subgroup (see Theorem 5). Using Theorem 4, we see that results of Kobayashi and Nagano in [3] give a classification of the reducible simple involutive Lie algebras. The analogy between Theorem 4 and the corollary to Theorem 5 indicates that a similar method of classification may work for the pseudo-hermitian symmetric spaces.

It is my pleasant duty to express my gratitude to Professors S. Kobayashi, J. A. Wolf and T. Nagano for their guidance and encouragement.

2. Fibration of affine symmetric spaces. Let $G$ be a Lie group and let $A_{1}, \cdots, A_{n}$ be subsets of $G$, each endowed with the induced topology. We say that $G=A_{1} \cdot A_{2} \cdots A_{n}$ topologically, if the map $\left(a_{1}, a_{2}, \cdots, a_{n}\right) \rightarrow a_{1} a_{2} \cdots a_{n}$ of $A_{1} \times A_{2} \times \cdots \times A_{n}$ into $G$ is a surjective homeomorphism.

Let $(\mathfrak{G}$ be the Lie algebra of $G$. A subset $B$ of $G$ is called an exponential set if there exist linearly independent linear subspaces $\mathfrak{b}_{1}, \mathfrak{b}_{2}, \cdots, \mathfrak{b}_{p}$ of $\mathfrak{G}$ such that $\left(v_{1}, v_{2}, \cdots, v_{p}\right) \rightarrow\left(\exp v_{1}\right)\left(\exp v_{2}\right) \cdots\left(\exp v_{p}\right)$ is a homeomorphism of $\mathfrak{b}_{1}+\mathfrak{b}_{2}+\cdots+$ $\mathfrak{b}_{p}$ onto $B$; if this is the case, we write $B=\operatorname{Exp}\left(\mathfrak{b}_{1}, \cdots, \mathfrak{b}_{p}\right)$. An exponential set $B=\operatorname{Exp}\left(\mathfrak{b}_{1}, \cdots, \mathfrak{b}_{p}\right)$ is said to be $S$-invariant, where $S$ is a subset of $G$, if each $\mathfrak{b}_{i}$ $(i=1, \cdots, p)$ is Ad $S$-invariant. It is clear that if $B$ is $S$-invariant then $s B s^{-1}=B$ for all $s \in S$.

Now, let $\theta$ be an involutive automorphism of $(5$. As a vector space, $(5$ decomposes 
into the $( \pm 1)$-eigenspaces of $\theta:\left(\mathfrak{G}=\mathfrak{G}^{+}+\mathfrak{G}^{-}\right.$. The subset $B=\operatorname{Exp}\left(\mathfrak{b}_{1}, \cdots, \mathfrak{b}_{p}\right)$ of $G$ is called a $(+)$-exponential set (with respect to $\theta$ ) if each $\mathfrak{b}_{i}(i=1, \cdots, p)$ is in $\mathfrak{G}^{+}$. The (-)-exponential sets of $G$ are defined in a similar fashion.

MaIN Lemma. Let $G$ be a connected Lie group and let $\Sigma$ be an involutive automorphism of $G$. Then

(1) $G$ contains a maximal compact subgroup $K$ stable under $\Sigma$, that is, $\Sigma K=K$, and

(2) there exist $K^{\Sigma}$-invariant (-)-exponential set $F$ and ( +)-exponential set $E$ of $G$ (with respect to $d \Sigma$ ) such that $G=K \cdot F \cdot E$ topologically.

The proof of this lemma is quite technical; we shall postpone it until next section.

COROLlary. Let $G$ be a Lie group. The fixed-point set of any involutive automorphism has only a finite number of connected components. If $G$ is furthermore simply-connected, the fixed-point set of any involutive automorphism is connected.

Let $\Sigma$ be any involutive automorphism of $G$, and denote the differential $d \Sigma$ of $\Sigma$ by $\sigma$. From the fact that $\Sigma \exp v=\exp \sigma v$, for all $v \in \mathbb{F}$, we conclude that the exponential sets $F$ and $E$ given in the Main Lemma are stable under $\Sigma$, and that $E$ is point-wise fixed by $\Sigma$ and the only fixed point of $\Sigma$ in $F$ is the identity $1 \in G$. Now, each element $g \in G$ decomposes uniquely into $g=k f e(k \in K, f \in F, e \in E)$, and $g \in G^{\Sigma}$ if and only if $k f e=\Sigma(k f e)=\Sigma(k) \Sigma(f) \Sigma(e)$. By uniqueness, we have $k \in K^{\Sigma}, f=1$, and hence $g \in K^{\Sigma} \cdot E$. It follows that

$$
G^{\Sigma}=K^{\Sigma} \cdot E \text {. }
$$

Since $E$ is connected, the number of connected components of $G^{\Sigma}$ is the same as that of $K^{\Sigma}$, which is finite thanks to the compactness of $K$. This proves the first assertion of the corollary. If $G$ is simply-connected, so is its maximal compact subgroup $K$. The second assertion now follows from (1) and the fact that $K^{\Sigma}$ is connected (see e.g. [2, p. 272, Theorem 7.2]).

The Main Lemma is designed to prove the following theorem, which has been stated with an erroneous proof by Berger in [1].

THEOREM 1. The symmetric space $(G / H, \Sigma)$ is fibered over a compact riemannian symmetric space $(K /(K \cap H), \Sigma \mid K)$ with fibers homeomorphic to a euclidean space. Furthermore, the symmetry induced by $\Sigma$ on $G / H$ is fiberpreserving.

Proof. From formula (1), each $h \in H$ is of the form $h=k e\left(k \in K^{\Sigma}, e \in E\right)$. Since $E$ is homeomorphic to a euclidean space and $1 \in E$, we may join $e$ to 1 by a path $e_{t}$ in $E$. Clearly, $k e_{t} \in H$; in particular, $k$ itself is in $H$. This shows that $H=(H \cap K) \cdot E$. We shall denote $H \cap K$ by $L$ for simplicity.

We now prove with Mostow that $G / H$ admits a fibering. It is well known that 
the space $K \times_{L} F=(K \times F) / \approx$ with $(k, f) \approx\left(k l, l^{-1} f l\right)$, together with the projection $[k, f] \rightarrow k L$, where $[k, f]$ is the equivalence class containing $(k, f)$, is a fiber bundle associated with the principal bundle $K \rightarrow K / L$.

Define a map $m: K \times F \rightarrow G / H$ by $m(k, f) \rightarrow k f H$. Then $m(k, f)=m\left(k^{\prime}, f^{\prime}\right)$ if and only if $(k, f) \approx\left(k^{\prime}, f^{\prime}\right)$. Thus $m$ induces a map $m: K \times_{L} F \rightarrow G / H$, which is one-to-one, and the following diagram is commutative:

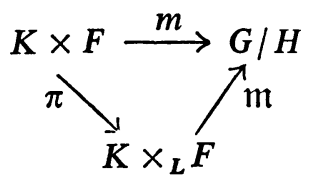

Since $m$ and the projection $\pi$ are open and continuous, $m$ is a homeomorphism. dIentifying $G / H$ with $K \times{ }_{L} F$, we get a fibration $G / H \stackrel{p}{\rightarrow} K / L$ with the projection $p: g H \rightarrow k L$, where $g=k f e$. Now, $p(\Sigma g H)=p(\Sigma k \Sigma f \Sigma e H)=(\Sigma k) L$ completes the proof of our theorem.

COROLlary 1. $\pi_{1}(G / H)$ is a finitely generated abelian group and $\pi_{0}\left(G^{\Sigma}\right)$ is a finite abelian group.

It has been shown by J. A. Wolf (Comment. Math. Helv. 37 (1962), 65-101; see p. 100) that the fundamental group of a riemannian symmetric space is abelian. Theorem 1 shows that any affine symmetric space is of the same homotopy type as a compact (riemannian) symmetric space, hence the first statement of the corollary. $\pi_{0}\left(G^{\Sigma}\right)$ is a quotient group of $\pi_{1}\left(G / G^{\Sigma}\right)$, hence abelian; it is also finite by the corollary of the Main Lemma.

COROLlaRY 2. If the symmetric space $(G / H, \Sigma)$ is effective, which we may always assume to be the case, then $G / H$ is compact if and only if $G$ is compact.

This follows directly from the fact that if $G / H$ is compact, then the maximal compact subgroup $K$ in the theorem acts transitively on the space $G / H$.

COROLlaRY 3. If $(G / H, \Sigma)$ is a riemannian symmetric space, then the fibration is topologically trivial. This shows, in particular, that any riemannian symmetric space is topologically the product of a compact symmetric space and a euclidean space.

Indeed, if $G / H$ is riemannian, then $H$ is compact. The relation $H=(H \cap K) \cdot E$ shows that $E=\{1\}$, i.e., $G=K \cdot F$ and $H=K \cap H=L$. A slight modification of the proof of the Main Lemma (see next section) will show that $G$ also decomposes into $G=K \cdot F^{\prime}$ topologically, where $F^{\prime}$ is a $K$-invariant exponential set. The fibering of $K \times{ }_{L} F^{\prime}$ over $K / L$ is easily seen to be topologically trivial. Now let $\Re$ be the Lie algebra of $K$; then, since $F$ and $F^{\prime}$ are $L$-invariant exponential sets, there are homeomorphisms $F \rightarrow \mathfrak{F} / \Re$ and $F^{\prime} \rightarrow \mathfrak{G} / \Omega$ commuting with the action of Ad $L$. It follows that $K \times_{L} F$ and $K \times{ }_{L} F^{\prime}$ are both bundle isomorphic to 
$K \times{ }_{L}(\mathfrak{b} / \mathfrak{\Omega})$. Thus $G / H$, being identified with $K \times_{L} F$, is fibered trivially over $K / L$.

Lemma A. Let $T$ be a compact connected abelian Lie group, and let $U$ be a closed connected subgroup of $T$. Then there exists a closed subgroup $W$ of $T$ such that $T=U+W$ (direct sum of groups).

Proof. We may assume that $T=V / \Gamma$, where $V$ is a vector group (i.e., euclidean $n$-space considered as additive Lie group; in fact we shall identify $V$ with the euclidean $n$-space), and $\Gamma$ is the group of lattice points in $V$. Let $\pi: V \rightarrow V / \Gamma$ be the natural projection. Then the connected component of identity $\tilde{U}$ of $\pi^{-1}(U)$ is a linear subspace of $V$. It follows that $\Gamma_{1}=\Gamma \cap \widetilde{U}$ is a pure subgroup of $\Gamma$. Since $\Gamma$ is free abelian, we have $\Gamma=\Gamma_{1}+\Gamma_{2}$, where $\Gamma_{2}$ is a subgroup of $\Gamma$, and the sum is direct. Now, $\pi(\widetilde{U})$ is compact; hence $\Gamma_{1}$ generates $\tilde{U}$ linearly. Let the linear subspace generated by $\Gamma_{2}$ be denoted by $\tilde{W}$. Then $V=\tilde{U}+\tilde{W}$, and $\tilde{W}$ projects onto a connected compact subgroup $W$ of $T$ satisfying the relation $T=U+W$. This proves Lemma A.

By a symmetric solvmanifold we mean an affine symmetric space $M=(G / H, \Sigma)$ with $G$ solvable.

Lemma B. Every compact symmetric solvmanifold is homeomorphic to a torus ( = cartesian product of circles).

Proof. Let $G / H$ be a compact symmetric manifold with $G$ solvable. Since the subgroups and quotient groups of a solvable group are again solvable, we may assume that $G / H=(G / H, \Sigma)$ is effective. It follows from Corollary 2 of Theorem 1 that $G$ is compact and hence abelian. Put $U=G_{0}^{\Sigma}$, the connected component of $G$, and $T=G$. Using the notation in the proof of Lemma A, we see that $\Sigma$ lifts into an automorphism $\tilde{\Sigma}$ of $V$ such that $\tilde{\Sigma} \Gamma=\Gamma$. Now $V$ decomposes into $V=V^{+}+V^{-}$ with respect to $\tilde{\Sigma}$. It is easily seen that $\Gamma_{1}=\Gamma \cap V^{+}$and $\Gamma_{2}$ may be taken to be $\Gamma \cap V^{-}$. Thus $T=U+W$ with $U=T_{0}^{\Sigma}$ and $\Sigma \mid W=-1$. Obviously,

$$
W^{\Sigma}=\left\{\pi(v) \mid 2 v \in \Gamma_{2}\right\}
$$

and $(H \cap W) \subset W^{\Sigma}$. Finally, $G / H=T / H=W /(W \cap H)$, the last space is visibly a torus.

THEOREM 2. Let $(G / H, \Sigma)$ be an affine symmetric space. We have

(a) if $G$ is nilpotent, then $G / H$ is homeomorphic to the product of a torus and $a$ euclidean space; and

(b) if $G$ is solvable then $G / H$ is covered finite times by the product of a torus and a euclidean space; if furthermore $H$ is connected, then $G / H$ is homeomorphic to the product of a torus and a euclidean space.

Proof. (a) If $G$ is nilpotent then the maximal compact subgroup $K$ in the 
Main Lemma is central. Hence $K \times_{L} F$ is the product of $F$ and $K / L$; the last space is a torus by Lemma $B$.

(b) It suffices to prove the second statement, since in general $H$ has only finite number of connected components. Again the maximal compact subgroup $K$ is abelian. If $H$ is connected, so is $L=H \cap K$. By Lemma A, the principal fiber bundle $K \rightarrow K / L$ is trivial, and consequently $K \times{ }_{L} F$, being associated to the above bundle, is a product bundle.

Among the 2-dimensional solvmanifolds, the euclidean plane $R^{2}$, the cylinder $S^{1} \times R$, the torus $T^{2}$, the Möbius strip, and the Klein bottle, only the last one is not symmetric. To see that the (open) Möbius strip is a symmetric solvmanifold we consider the group of motions in $R^{2}$, which may be considered as the set $\left\{\left(e^{i t}, a, b\right) \mid t, a\right.$, and $b$ are real numbers $\}$ with multiplication defined by $\left(e^{i t}, a, b\right)\left(e^{i u}, c, d\right)=\left(e^{i(t+u)}, c \cos t-d \sin t+a, c \sin t+c \cos t+b\right)$. Denote this group by $G$. The map $\left(e^{i t}, a, b\right) \rightarrow\left(e^{-i t},-a, b\right)$ is an involutive automorphism, to be denoted by $\Sigma$. Then $G / G^{\Sigma}$ gives the open Möbius strip. This example shows that the first statement in (b) of Theorem 2 is, in a certain sense, best possible.

Finally, we remark that if $G / H$ is an affine symmetric space for which $G$ is semi-simple and centerless, then the fibers in Theorem 1 are totally geodesic as one can see easily from the proof of the Main Lemma.

3. Proof of the Main Lemma. We begin this section with a few lemmata we shall need later.

LEMMA C. Let $G$ be a connected Lie group with an involutive automorphism $\Sigma$. Let $V$ be a closed normal vector subgroup of $G$ with $\Sigma V=V$, such that $G / V$ is compact. Then there exists a compact subgroup $L$ of $G$ which projects isomorphically onto $G / L$ and $\Sigma L=L$.

Proof. Consider the extension $V \rightarrow G \rightarrow G / V$ of a compact Lie group, $G / V$, by a vector group, $V$. By a theorem of $\mathrm{K}$. Iwasawa, there exists a section which is a subgroup; furthermore, any two such sections are conjugate by an element of $V$. Thus $G$ can be decomposed into the semi-direct product, $G=V K$, of $V$ and a compact subgroup $K$ which projects isomorphically onto $G / V$. Let $\mathfrak{G}=\mathfrak{B}+\mathfrak{R}$ be the corresponding decomposition of the Lie algebras. We shall show that if $X \in \mathfrak{V}$ is such that $\operatorname{Ad}(\exp X) \Re \subset \Re$, then $\exp t X$ is in the centralizer of $K$ in $G$ for any real number $t$. Indeed, for an arbitrary $Y \in \Re$, we have $\operatorname{Ad}(\exp X) Y$ $=e^{\operatorname{ad} X} Y=Y+[X, Y]$, since $\mathfrak{B}$ is an abelian ideal in $\mathfrak{G}$ and $[X, Y] \in \mathfrak{P}$. If $\operatorname{Ad}(\exp X) \Re \subset \Re$, then $[X, Y]$ is also in $\Re$. Thus $[X, Y]=0$. Since $Y \in \Re$ is arbitrary, we have $[t X, \Omega]=0$, i.e., $\exp t X$ centralizes $K$ in view of the connectedness of $K$.

From the conjugacy of the sections, we have $\Sigma K=g K g^{-1}=g_{-} g_{+} K_{g_{+}^{-1}} g_{-}^{-1}$, where $V=V^{+}+V^{-}$according to $\Sigma, g=g_{-} g_{+}, g_{+} \in V^{+}$and $g_{-} \in V^{-}$. Now $\Sigma \Sigma K=K$ and $g_{+}$commutes with $g_{-}$; it follows that $g_{+}^{2} K g_{+}^{2}=K$. Since $g_{+}^{2}$ $=\exp X$ for some $X$ in $\mathfrak{B}$, from what we proved in the preceding paragraph we 
conclude that $g_{+}$centralizes $K$ and hence $\Sigma K=g_{-} K_{-}^{-1}$. It is now clear that $L=g_{-}^{1 / 2} \mathrm{Kg}_{-}^{-1 / 2}$ is the required compact subgroup.

Lemma D. Let $G$ be a connected Lie group. If $G$ is not semi-simple, then $G$ contains either a nontrivial central toroidal subgroup or a nontrivial normal closed vector group stable under all automorphisms of $G$.

Proof. It is obvious that the radical of $G$ contains a nontrivial abelian subgroup $A$ of $G$ stable under all automorphisms of $G$. If $\bar{A}$, the closure of $A$, is a vector group, we are through; otherwise, let $T$ be the maximal toroidal subgroup of $\bar{A}$. Then $T$ is stable under the group of automorphisms of $G$. It remains to show that $T$ is central. For each $g \in G$, let $\alpha_{g}$ denote the inner automorphism of $G$ defined by $g$. Then the map $g \rightarrow \alpha_{g} \mid T$ of $G$ into the group of automorphisms of $T$ is a (continuous) homomorphism. Since the latter group is easily seen to be discrete and since $G$ is connected, $\alpha_{g} \mid T$ is trivial for all $g \in G$. This proves that $T$ is central and hence the lemma.

Now, let $G$ be a semi-simple Lie groupe with Lie algebra (5.Then the Killing form ( = the fundamental bilinear form) $B$ of $\mathbb{G}$ is nondegenerate. By a Cartan decomposition of $\mathfrak{G}$ is meant a direct sum decomposition $\mathfrak{G}=\mathfrak{K}+\mathfrak{P}$, where $\mathfrak{K}$ is a subalgebra and $\mathfrak{P}$ is a linear subspace of $\mathfrak{G}$ such that the Killing form of $\mathfrak{b}$ is negative (resp. positive) defined on $\mathfrak{A}$ (resp. on $\mathfrak{P}$ ), and the map $\theta: X+Y \rightarrow X-Y$ $(X \in \mathfrak{R}, Y \in \mathfrak{P})$ is an automorphism of $G$. Such an automorphism is called a *-automorphism. In general, not every automorphism of $\mathfrak{G}$ extends to an automorphism of $G$, unless $G$ is simply connected. However, it is known that every *-automorphism of $\mathfrak{G}$ can be extended to an automorphism of $G$, whether $G$ is simply connected or not. To see this, we consider the corresponding Cartan decomposition $\mathfrak{F}=\mathfrak{K}+\mathfrak{P}$ of the given *-automorphism $\theta$. Let $\tilde{G}$ denote the universal covering group of $G$ and $\widetilde{K}$ the connected subgroup generated by $\Omega$ in $\widetilde{G}$. It follows from the fact that $\operatorname{Ad}(\tilde{G}) / \operatorname{Ad}(\tilde{K})$ is simply-connected that the centrum of $\tilde{G}$ is contained in $\widetilde{K}$. Extend $\theta$ to an automorphism $\tilde{\Theta}$ of $\widetilde{G}$. Then $\Theta$ leaves the centrum of $G$ pointwise fixed. Since the kernel of the projection $\tilde{G} \rightarrow G$ is central $\tilde{\Theta}$ induces an automorphism $\Theta$ of $G$ which extends $\theta$.

Finally we recall a theorem of Mostow (Memoirs Amer. Math. Soc., No. 14, 1955) which states:

Let $G$ be a connected real semi-simple Lie group, and let $\mathfrak{G}=\Omega+\mathfrak{P}$ be a Cartan decomposition of the Lie algebra of $G$. Let $\mathfrak{E}$ be a linear subspace of $\mathfrak{P}$ such that $[X,[X, Y]] \in \mathfrak{E}$ for all $X, Y$ in $\mathfrak{E}$. Let $\mathfrak{F}=\{X \mid B(X, \mathfrak{E})=0, X \in \mathfrak{P}\}$. Then $G$ decomposes topologically into $K \cdot F \cdot E$ where $K$ is the analytic subgroup determined by $\mathfrak{K}, F=\exp \mathfrak{F}$, and $E=\exp \mathfrak{E}$.

It should be remarked that $F$ and $E$ are both exponential sets in the sense that the exponential maps of $\mathfrak{F}$ onto $F$ and of $\mathfrak{E}$ onto $E$ are homeomorphisms. Let $\theta$ denote the *-automorphism associated with the given Cartan decomposition 
$\mathfrak{G}=\mathfrak{R}+\mathfrak{P}$, and let $\Theta$ denote the automorphism induced on $G$ by $\theta$. Since $\theta=-1$ on $\mathfrak{E}$ and $\mathfrak{F}$, we conclude that $K=G^{\boldsymbol{\Theta}}$.

We are ready to prove the Main Lemma. We shall proceed by induction on the dimension of $G$.

Case I. $G$ is semi-simple.

It has been proved by various authors $\left({ }^{1}\right)$ that for any given involutive automorphism $\Sigma$ of $G$, there exists a $*$-automorphism $\theta$ of $\mathfrak{G}$ which commutes with $\sigma=d \Sigma$. Let $\mathbb{G}=\mathfrak{\Omega}_{1}+\mathfrak{P}$ be the Cartan decomposition corresponding to $\theta$. Then $\mathfrak{R}_{1}$ and $\mathfrak{P}$ are both invariant under $\sigma$. Thus $\mathfrak{P}=\mathfrak{P}^{+}+\mathfrak{P}^{-}$with respect to $\sigma$. Since the Killing form $B$ of $\mathfrak{G}$ is definite on $\mathfrak{P}$ and since $\sigma$ is an automorphism, we conclude that $\mathfrak{P}^{-}=\left\{X \in \mathfrak{P} \mid B\left(X, \mathfrak{P}^{+}\right)=0\right\}$. Clearly, $\left[\mathfrak{P}^{+},\left[\mathfrak{P}^{+}, \mathfrak{P}^{+}\right]\right] \subset \mathfrak{P}^{+}$. The theorem of Mostow which we quoted just now shows that $G=K_{1} \cdot F^{\prime} \cdot E$ topologically, where $K_{1}$ is the analytic subgroup generated by $\Re_{1}, F^{\prime}=\exp \left(\mathfrak{P}^{-}\right)$ and $E^{\prime}=\exp \left(\mathfrak{P}^{+}\right)$. Notice that $K_{1}$ is stable under $\Sigma$.

Let $\Theta$ denote the automorphism of $G$ obtained by extending the *-automorphism $\theta$. Then, as we remarked before, $G^{\Theta}=K_{1}$. An element $g$ of $G$ is in $K_{1}^{\Sigma}$ if, and only if, $\Theta g=g$ and $\Sigma g=g$. It is obvious that an element $X$ in $\mathscr{G}$ is in $\mathfrak{P}^{-}$if, and only if, $\theta X=-X$, and $\sigma X=-X$. Thus the relations

$$
\begin{aligned}
& \theta \operatorname{Ad}(g) X=\operatorname{Ad}(\Theta g) \theta X, \text { and } \\
& \sigma \operatorname{Ad}(g) X=\operatorname{Ad}(\Sigma g) \sigma X
\end{aligned}
$$

show that $\mathfrak{P}^{-}$is $K_{1}^{\Sigma}$-invariant, i.e., $\operatorname{Ad}\left(K_{1}^{\Sigma}\right) \mathfrak{P}^{-} \subset \mathfrak{P}^{-}$. Similarly, $\mathfrak{P}^{+}$is also $K_{1}^{\Sigma}$-invariant.

The group $K_{1}$ is in general not compact. Since its Lie algebra $\Omega_{1}$ is compactly imbedded, $K_{1}=K \times V$, where $K$ is a maximal compact subgroup of $K_{1}$ and $V$ is a vector group. Since $\Sigma K_{1}=K_{1}$, and since the decomposition $K_{1}=K \times V$ is direct, we have $\Sigma K=K$ (indeed, $K$ is the only maximal compact subgroup of $K_{1}$ ). It is obvious that $K$ is a maximal compact subgroup of $G$ as well.

Decompose $V$ into $V^{+}+V^{-}$with respect to $\Sigma$. $V^{+} \subset K_{1}^{\Sigma}$ shows that $\operatorname{Ad}\left(V^{+}\right) \mathfrak{P}^{-} \subset \mathfrak{B}$, i.e., $V^{+}\left(\exp \mathfrak{B}^{-}\right) V^{+}=V^{+} F^{\prime} V^{+}=F^{\prime}$. Thus

$$
G=K_{1} \cdot F^{\prime} \cdot E^{\prime}=K \cdot V^{-} \cdot V^{+} \cdot F^{\prime} \cdot E^{\prime}=K \cdot V^{-} \cdot F^{\prime} \cdot V^{+} \cdot E^{\prime}=K \cdot F \cdot E,
$$

where $F=V^{-} \cdot F^{\prime}=\operatorname{Exp}\left(\mathfrak{Y}^{-}, \mathfrak{P}^{-}\right)$is a $(-)$-exponential set, and $E=V^{+} \cdot E^{\prime}$ $=\operatorname{Exp}\left(\mathfrak{B}^{+}, \mathfrak{P}^{+}\right)$is a $(+)$-exponential set. Notice that $F$ and $E$ are $K^{\Sigma}$-invariant.

Case II. $G$ contains a nontrivial central toroidal subgroup $T$.

We may assume that $\Sigma T=T$. Put $\hat{G}=G / T$ and let $\pi: G \rightarrow \hat{G}$ be the natural projection. Since $T$ is stable under $\Sigma, \Sigma$ induces an involutive automorphism $\hat{\Sigma}$ of $\hat{G}$. By induction hypothesis, we have

$$
\hat{G}^{\prime}=\cdot \hat{K} \cdot \sim \hat{F} \cdot \hat{E},
$$

(1) See e.g. [3], [6] or the Appendix of this paper. 
where $\hat{K}$ is a maximal compact subgroup in $\hat{G}$ stable under $\hat{\Sigma}, \hat{F}=\operatorname{Exp}\left(\hat{\mho}_{1}, \cdots, \hat{\mho}_{p}\right)$, $\left(\hat{\mathfrak{F}}_{i} \subset \hat{\mathfrak{G}}^{-}\right), \hat{E}=\operatorname{Exp}\left(\hat{\mathfrak{E}}_{1}, \cdots, \hat{\mathbb{E}}_{q}\right),\left(\hat{\mathfrak{E}}_{j} \subset \hat{\mathfrak{G}}^{+}\right)$, and $\hat{F}$ and $\hat{E}$ are $\hat{K}^{\hat{\Sigma}}$-invariant. Since $T$ is compact, $K=\pi^{-1}(\hat{K})$ is a maximal compact subgroup of $G$, and $K$ is obviously stable under $\Sigma$.

Let $\mathfrak{M}_{i}=\pi^{-1}\left(\hat{\mathfrak{\Im}}_{i}\right)$ and $\mathfrak{N}_{j}=\pi^{-1}\left(\widehat{\mathfrak{E}}_{j}\right)$. It follows from the facts $d \pi($ Ad $g) X$ $=\operatorname{Ad}(\pi g) d \pi X$ and $\pi \Sigma=\hat{\Sigma} \pi$ that $\mathfrak{M}_{i}$ and $\mathfrak{N}_{j}$ are $K^{\Sigma}$-invariant and are stable under $\Sigma$. Let $\mathfrak{I}$ denote the Lie algebra of $T$. Then $\mathfrak{I}=\mathfrak{I}^{+}+\mathfrak{T}^{-}$with respect to $\sigma$. Now $d \pi\left(\mathfrak{M}_{i}^{+}\right)=0$ shows that $\mathfrak{M}_{i}^{+}=\mathfrak{I}^{+}$; similarly $\mathfrak{R}_{j}^{-}=\mathfrak{I}^{-}$, where $\mathfrak{M}_{i}^{+}, \mathfrak{M}_{i}^{-}, \mathfrak{N}_{j}^{+}$ and $\mathfrak{N}_{j}^{-}$are with respect $\sigma(i=1, \cdots, p ; j=1, \cdots, q)$. It is obvious that $\mathfrak{I}^{+}$and $\mathfrak{I}^{-}$are $K^{\Sigma}$-invariant, for $\mathfrak{I}$ is central. As $K^{\Sigma}$ is compact, $\operatorname{Ad} K^{\Sigma}$ is fully reducible. Let $\mathfrak{F}_{i}$ be the $K^{\Sigma}$-invariant supplement of $\mathfrak{I}^{-}$in $\mathfrak{M}_{i}^{-}$and let $\mathfrak{E}_{j}$ be the $K^{\Sigma}$-invariant supplement of $\mathfrak{I}^{+}$in $\mathfrak{N}_{j}^{+}$. Thus $\mathfrak{M}_{i}=\mathfrak{I}^{+}+\mathfrak{I}^{-}+\mathfrak{F}_{i}$ with $\mathfrak{F}_{i} \subset \mathfrak{G}^{-}$, and $\mathfrak{N}_{j}$ $=\mathfrak{T}^{+}+\mathfrak{T}^{-}+\mathfrak{E}_{j}$ with $\mathfrak{E}_{j} \subset \mathfrak{G}^{+}$. Notice that $d \pi: \mathfrak{F}_{i} \rightarrow \hat{\mathscr{F}}_{i}$ and $d \pi: \mathfrak{E}_{j} \rightarrow \hat{\mathfrak{E}}_{j}$ are bijective. It follows from the commutative diagrams
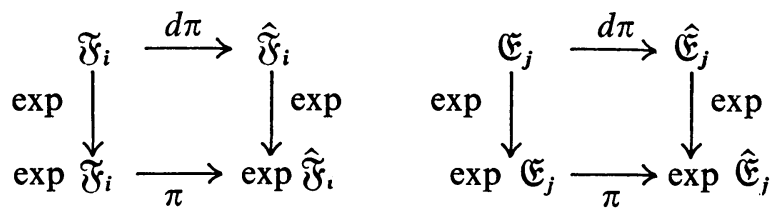

and the fact that $\hat{F}=\operatorname{Exp}\left(\widehat{\mathscr{F}}_{1}, \cdots, \hat{\mathfrak{F}}_{p}\right)$ and $\hat{E}=\operatorname{Exp}\left(\hat{\mathfrak{E}}_{1}, \cdots, \hat{\mathfrak{F}}_{q}\right)$ are exponential sets that $\pi$ is bijective on $F=\exp \mathfrak{F}_{1} \exp \mathfrak{F}_{2} \cdots \exp \mathfrak{F}_{p}$ and $E=\exp \mathfrak{E}_{1} \exp \mathfrak{E}_{2} \cdots \exp \mathfrak{E}_{q}$. It is now clear that $F$ and $E$ are exponential sets.

Since $\hat{G}=\pi(K F E)$, we have $G=K F E T=K T F E=K F E$. It remains to show that $G=K \cdot F \cdot E$ topologically. Define a map $\mu: K \times F \times E \rightarrow G$ by $\mu(k, f, e)$ $=k f e$. Let $g \in G$ be an arbitrary element. Then $g=k f e$. Now $\pi(g)=\hat{k} \hat{f} \hat{e}$ determines $\hat{k}, \hat{f}, \hat{e}$ uniquely; hence $f, e$, and consequently $k$, are determined uniquely by $g$. This proves that $\mu$ is bijective. Finally, from the fact that $\mu$ is a bijective continuous map of a manifold $K \times F \times E$ onto another manifold of the same dimension, we conclude that $\mu$ is a homeomorphism.

Case III. $G$ contains a nontrivial normal closed vector subgroup $V$.

Again we may assume that $V$ is stable under $\Sigma$. Let $\hat{G}=G / V, \pi: G \rightarrow \hat{G}$ the natural projection and let $\hat{\Sigma}$ be the induced automorphism on $\hat{G}$.

By induction hypothesis $\hat{G}=\hat{K} \cdot \hat{F} \cdot \hat{E}$. Now, $\pi^{-1}(\hat{K})=L$ is a closed subgroup of $G$ stable under $\Sigma$, and $L$ contains $V$ as a normal subgroup. Applying Lemma $C$, we obtain a maximal compact subgroup $K$ of $L$, stable under $\Sigma$ such that $\pi \mid K$ is an isomorphism of $K$ onto $\hat{K}$. As $\hat{K}$ is maximal compact in $\hat{G}, K$ is maximal compact in $G$.

Let $\hat{\mathfrak{F}}_{i}$ and $\hat{\mathfrak{G}}_{j}$ be defined as in Case II. Again we let $\mathfrak{M}_{i}=(d \pi)^{-1} \hat{\mathfrak{F}}_{i}$ and $\mathfrak{N}_{j}=(d \pi)^{-1} \widehat{\mathfrak{E}}_{j}$. Then $\mathfrak{M}_{i}$ and $\mathfrak{N}_{j}$ are stable under $\Sigma$. From the facts

(1) an element $g$ in $G$ is in $K^{\Sigma}$ if and only if $\pi g \in(\hat{K})^{\hat{\Sigma}}$, 
(2) $d \pi(\operatorname{Ad} g) X=\operatorname{Ad}(\pi g) d \pi(X)$, and

(3) $\hat{\mathfrak{F}}_{i}$ and $\hat{\mathfrak{E}}_{j}$ are $\operatorname{Ad}(\hat{K})^{\hat{\Sigma}^{2}}$-invariant $(i=1, \cdots, p ; j=1, \cdots, q)$, we conclude that $\mathfrak{M}_{i}$ and $\mathfrak{N}_{j}$ are $K^{\Sigma}$-invariant.

A similar argument as the one used in Case II shows that

$$
\mathfrak{M}_{i}=\mathfrak{B}^{+}+\mathfrak{B}^{-}+\mathfrak{F}_{i}^{\prime} \text {, and } \mathfrak{N}_{j}=\mathfrak{B}^{+}+\mathfrak{B}^{-}+\mathfrak{E}_{j}^{\prime},
$$

where $\mathfrak{F}_{i}^{\prime} \subset \mathfrak{G}^{-}$and $\mathfrak{E}_{j}^{\prime} \subset \mathfrak{G}^{+}$and $d \pi$ is bijective on $\mathfrak{F}_{i}^{\prime}$ and on $\mathfrak{E}_{j}^{\prime}$.

Now $G=K F^{\prime} E^{\prime} V=K F^{\prime} V E^{\prime}=K F^{\prime} V^{-} V^{+} E^{\prime}=K F E$, where $F=F^{\prime} V^{-}$, and $E=V^{+} E^{\prime}$. It remains to show that $G=K \cdot F^{\prime} \cdot V^{-} \cdot V^{+} \cdot E^{\prime}$ topologically. Since $V=V^{-} \cdot V^{+}$, we need only show that $G=K \cdot F^{\prime} \cdot V \cdot E^{\prime}$. Define a map $\mu: K \times F^{\prime} \times V \times E^{\prime} \rightarrow G$ by $\mu(k, f, v, e)=k f v e$. It is obvious that if $k_{1} f_{1} v_{1} e_{1}$ $=k_{2} f_{2} v_{2} e_{2}$ then $\pi\left(k_{1}\right)=\pi\left(k_{2}\right), \pi\left(f_{1}\right)=\pi\left(f_{2}\right)$ and $\pi\left(e_{1}\right)=\pi\left(e_{2}\right)$; consequently $k_{1}=k_{2}, f_{1}=f_{2}, e_{1}=e_{2}$, and hence $v_{1}=v_{2}$. It follows that $\mu$ is a homeomorphism (see the proof of Case II), and $F=\operatorname{Exp}\left(\mathfrak{F}_{1}, \cdots, \mathfrak{F}_{p}, \mathfrak{B}^{-}\right)$and $E=\operatorname{Exp}\left(\mathfrak{V}^{+}, \mathfrak{E}_{1}, \cdots, \mathfrak{E}_{q}\right)$.

The Main Lemma now follows from Lemma $D$, and the proof is thus complete.

4. Semi-simple involutive Lie algebras. A pair $(\mathfrak{5}, \sigma)$, consisting of a (real) Lie algebra $(\mathfrak{5}$ and an involutive automorphism $\sigma$ of $G$, is called an involutive Lie algebra (abbreviated iLa) if no nontrivial ideal of $\mathfrak{b}$ is left pointwise fixed by $\sigma$. Now $(\mathfrak{G}$, as a vector space, has a direct sum decomposition (called the canonical decomposition)

$$
\mathfrak{G}=\mathfrak{H}+\mathfrak{M},
$$

where $\mathfrak{H}$ is the $(+1)$-eigenspace and $\mathfrak{M}$ the $(-1)$-eigenspace of $\sigma$. Notice that $\mathfrak{H}$ is a subalgebra, $[\mathfrak{H}, \mathfrak{M}] \subset \mathfrak{M}$, and $[\mathfrak{M}, \mathfrak{M}] \subset \mathfrak{H}$. Let $(\mathfrak{G}, \sigma)$ and $\left(\mathfrak{G}^{\prime}, \sigma^{\prime}\right)$ be two iLa's. Their direct sum is defined to be the iLa $\left(\mathfrak{5}+\left(\mathfrak{5}^{\prime}, \sigma \times \sigma^{\prime}\right)\right.$, where $\left(\sigma \times \sigma^{\prime}\right)(X, Y)$ $=\left(\sigma X, \sigma^{\prime} Y\right)$ for all $(X, Y)$ in $\left(\mathfrak{b}+\mathfrak{G}^{\prime}\right.$. The iLa's $(\mathfrak{G}, \sigma)$ and $\left(\mathfrak{F}^{\prime}, \sigma^{\prime}\right)$ are said to be isomorphic, if there exists a Lie algebra isomorphism $f: \mathfrak{G}_{\rightarrow} \rightarrow \mathfrak{G}^{\prime}$ such that $f \circ \sigma=\sigma^{\prime} \circ f$.

Let $G$ be the connected, simply-connected Lie group whose Lie algebra is $\mathfrak{G}$. Let $H=G^{\Sigma}$ be the subgroup of fixed points of $\Sigma$, where $\Sigma$ is the analytic automorphism of $G$ generated by $\sigma$. It is clear that $H$ is the analytic subgroup generated by $\mathfrak{H}$. The affine symmetric space $(G / H, \Sigma)$ is, by the corollary of the Main Lemma, simply-connected; to be called the affine symmetric space associated with the iLa $(\mathfrak{G}, \sigma)$. Isomorphic iLa's define affinely equivalent associated symmetric spaces.

Conversely, every affine symmetric space $\left(G^{*} / H^{*}, \Sigma^{*}\right)$ gives rise to an iLa $(\mathfrak{G}, \sigma)$, where $\mathfrak{G}$ is the Lie algebra of $G^{*}$ and $\sigma=d \Sigma^{*}$. The affine symmetric space $(G / H, \Sigma)$ associated with $(\mathfrak{5}, \sigma)$ is a universal covering manifold of $\left(G^{*} / H^{*}, \Sigma^{*}\right)$. Inasmuch as the local properties of a Lie group are determined completely by its Lie algetra the local properties of an affine symmetric space are completely described by its iLa. This justifies the study of iLa's. 
Let $\mathfrak{5}=\mathfrak{H}+\mathfrak{M}$ be the canonical decomposition of an iLa $(\mathfrak{5}, \sigma)$. The iLa will be called pseudo-riemannian, if there exists an ( $\operatorname{ad} \mathfrak{S})$-invariant nondegenerate quadratic form $Q$ on $\mathfrak{M}$; almost complex, if there exists an (ad $\mathfrak{H})$-invariant complex structure on $\mathfrak{M}$; pseudo-hermitian, if it is almost complex and, with respect to this complex structure, there exists an $(\mathrm{ad} \mathfrak{T})$-invariant nondegenerate hermitian form on $\mathfrak{M}$. To express that $(\mathfrak{F}, \sigma)$ is endowed with an invariant pseudoriemannian (-hermitian) metric $Q$, we often write $(\mathfrak{5}, \sigma, Q)$ for $(\mathfrak{5}, \sigma)$.

The following is known (see e.g. [1]).

Proposition. The affine symmetric space associated $\left(^{2}\right)$ with the iLa $(\mathfrak{5}, \sigma)$ has an invariant pseudo-riemannian, complex or semi-kählerian structure according as the iLa is pseudo-riemannian, almost complex or pseudo-hermitian.

An $\mathrm{iLa}(\mathfrak{5}, \sigma)$ is called semi-simple (resp. simple, etc.) if the Lie algebra $\mathfrak{G}$ is semi-simple (resp. simple, etc.). We shall agree, once for all, to denote the Killing form of $(\mathfrak{G}$ by $B$. It is obvious that if $(\mathfrak{5}, \sigma)$ is semi-simple, $(\mathfrak{5}, \sigma, B)$ is a pseudoriemannian iLa.

As we remarked before (see $\S 3$ ), if the $\operatorname{iLa}((5, \sigma)$ is semi-simple, then there exists a $*$-automorphism $\theta$ of $(\mathfrak{5}$ which commutes with $\sigma$. The corresponding Cartan decomposition $\mathfrak{G}=\mathfrak{R}+\mathfrak{P}$ is called a Cartan decomposition of the iLa $(5, \sigma)$.

LEMMA E. Let $\mathfrak{G}=\mathfrak{H}+\mathfrak{M}$ be the canonical decomposition of a semi-simple iLa $(\mathfrak{G}, \sigma)$. Then $\operatorname{ad}_{\mathfrak{G}} \mathfrak{H}$ is completely reducible and hence $\mathfrak{H}$ is reductive.

Proof. Let $\mathfrak{G}=\mathfrak{R}+\mathfrak{P}$ be a Cartan decomposition of $(\mathfrak{G}, \sigma)$. Then $\mathfrak{H}=\mathfrak{H} \cap \mathfrak{R}+\mathfrak{G} \cap \mathfrak{P}$. It is well known (and it is easy to show) that there exists a basis of $\mathfrak{G}$, with respect to which $\operatorname{ad}_{\mathfrak{G}} \mathfrak{P}\left(\right.$ resp. $\left.\operatorname{ad}_{\mathfrak{G}} \mathfrak{R}\right)$ is represented by a set of symmetric (resp. skew-symmetric) matrices. Thus $\mathrm{ad}_{\mathfrak{G}} \mathfrak{H}$ is represented by a selfadjoint family of matrices, hence completely reducible.

LEMMA $\mathrm{F}$. For any $\left(\operatorname{ad}_{\mathfrak{M}} \mathfrak{H}\right)$-invariant quadratic form $Q$ on $\mathfrak{M}$, we have, for arbitrary elements $X, Y, Z, T$ in $\mathfrak{M}$,

$$
Q([[X, Y], Z], T)=Q([[Z, T], X], Y) .
$$

Proof. This is a particular case of Lemma 12.4 of $[2$, p. 68], whose proof consists of a simple algebraic manipulation.

Let $(\mathfrak{G}, \sigma, Q)$ be a pseudo-riemannian iLa. A linear subspace $\mathfrak{U}$ of $\mathfrak{M}$ is called a $Q$-subspace, if the restriction of $Q$ on $\mathfrak{U}$ is nondegenerate. The iLa $(\mathfrak{G}, \sigma, Q)$ is called $Q$-irreducible, if $\mathfrak{M}$ contains no proper nontrivial (adS)-invariant $Q$ subspaces.

LEMMA G. Let $(5, \sigma, Q)$ be a pseudo-riemannian semi-simple iLa. Then

(2) This implies that $G^{\Sigma}=H$ is connected. 
every (ad $\mathfrak{G})$-invariant $Q$-subspace $\mathfrak{U}$ of $\mathfrak{M}$ admits an (ad $\mathfrak{H})$-invariant supplementary $Q$-subspace $\mathfrak{B}$ in $\mathfrak{M}$ such that $\mathfrak{U}$ and $\mathfrak{B}$ are orthogonal under $Q$.

Proof. Let $\mathfrak{U}^{\perp}=\{X \in \mathfrak{M} \mid Q(X, \mathfrak{U})=0\}$. Then $\mathfrak{M}=\mathfrak{U}+\mathfrak{U}^{\perp}$, since $Q$ is nondegenerate on $\mathfrak{U}$. Indeed, for an arbitrary $A \in \mathfrak{M}$, there exists a unique element $A^{\prime}$ in $\mathfrak{U}$ such that $Q\left(A^{\prime}, Z\right)=Q(A, Z)$ for all $Z$ in $\mathfrak{U}$. It follows that $A^{\prime \prime}=A-A^{\prime}$ is in $\mathfrak{U}^{\perp}$ and $A=A^{\prime}+A^{\prime \prime}$ gives the decomposition. Now, $\mathfrak{U}$ is invariant ( $=\operatorname{ad} \mathfrak{H}$ invariant), so is $\mathfrak{U}^{\perp}$. Since $\mathfrak{U} \cap \mathfrak{U}^{\perp}=0$, the lemma follows.

In the canonical decomposition $\mathfrak{G}=\mathfrak{H}+\mathfrak{M}$ of an iLa, we say that a linear subspace $\mathfrak{U}$ of $\mathfrak{M}$ splits off, if $[\mathfrak{U}, \mathfrak{U}]+\mathfrak{U}$ is an ideal of $\mathfrak{b}$.

LEMMA H. In a semi-simple pseudo-riemannian iLa $(\mathfrak{5}, \sigma, Q)$, every invariant $Q$-subspace of $\mathfrak{M}$ splits off. In particular, every simple iLa is B-irreducible, where $B$ is the Killing form of $(5$.

Proof. Let $\mathfrak{U}$ be a $Q$-subspace in $\mathfrak{M}$. By Lemma $\mathrm{G}$, there exists an invariant supplement space, $\mathfrak{B}$, of $\mathfrak{U}$ in $\mathfrak{M}$ such that $\mathfrak{B} \perp_{Q} \mathfrak{U}$ (read : $\mathfrak{U}$ and $\mathfrak{B}$ are orthogonal under $Q$ ). Put $\mathfrak{I}=\mathfrak{U}+[\mathfrak{U}, \mathfrak{U}]$. A routine computation shows that $\mathfrak{I}$ is a subalgebra of $\mathfrak{G}$ and $[\mathfrak{I}, \mathfrak{H}] \subset \mathfrak{I}$. By Jacobi identity $[\mathfrak{V},[\mathfrak{U}, \mathfrak{U}]]=[\mathfrak{U},[\mathfrak{V}, \mathfrak{U}]]$. The left-hand side of the equality is contained in $\mathfrak{B}$, and the right-hand side belongs to $\mathfrak{U}$; hence $[\mathfrak{B},[\mathfrak{U}, \mathfrak{U}]]=0$. Now, for arbitrary elements $X \in \mathfrak{U}, Y \in \mathfrak{B}$, and $Z, T$ in $\mathfrak{M}$, we have, by (2), $Q([Z,[X, Y]], T)=-Q([Y,[T, Z]], X)=0$, since $[Y,[T, Z]]$ belongs to $\mathfrak{B}$ and $\mathfrak{B} \perp_{Q} \mathfrak{U}$. It follows from the nondegeneracy of $Q$ that $[Z,[X, Y]]=0$, for all $Z \in \mathfrak{M}$. Finally, $\operatorname{ad}_{\mathfrak{M}} \mathfrak{H}$ is faithful and $[X, Y] \in \mathfrak{S}$ prove that $[X, Y]=0$, and the lemma follows.

In the proof of the preceding lemma, we have also established that if $\mathfrak{U}$ and $\mathfrak{B}$ are invariant supplementary $Q$-subspaces of $\mathfrak{M}$ then $[\mathfrak{U}, \mathfrak{P}]=0$.

Let $(\mathfrak{5}, \sigma)$ be a semi-simple iLa. Decompose $\mathfrak{G}$ into the direct sum of simple ideals:

$$
\mathfrak{G}=\mathfrak{I}_{1}+\mathfrak{I}_{2}+\cdots+\mathfrak{I}_{n} .
$$

Since $\sigma$ permutes the $\mathfrak{I}_{j}$ 's among themselves, the iLa $(\mathfrak{G}, \sigma)$ admits a direct sum decomposition

$$
\left((\mathfrak{G}, \sigma)=\left(\mathfrak{G}_{1}, \sigma\right)+\cdots+\left(\mathfrak{G}_{p}, \sigma\right)+\left(\mathfrak{L}_{1}, \sigma\right)+\cdots+\left(\mathfrak{L}_{q}, \sigma\right),\right.
$$

where $\mathfrak{G}_{j}=\mathfrak{I}_{k}$ with $\sigma\left(\mathfrak{I}_{k}\right)=\mathfrak{I}_{k}$, and $\mathfrak{I}_{j}=\mathfrak{I}_{k}+\mathfrak{I}_{m}$ with $\sigma\left(\mathfrak{I}_{k}\right)=\mathfrak{I}_{m}$. It is not hard to see that iLa $\left(\mathfrak{L}_{i}, \sigma\right)$ isomorphic to an iLa of the form $(\mathfrak{I}+\mathfrak{I}, \tau)$ where $\mathfrak{I}$ is a simple Lie algebra and $\tau(X, Y)=(Y, X)$ for all $(X, Y)$ in $\mathfrak{I}+\mathfrak{I}$. The study of semi-simple iLa's is thus reduced to that of simple ones.

It should be remarked that even for a simple iLa, the representation $\operatorname{ad}_{\mathfrak{M i}} \mathfrak{H}$ may very well be reducible. If this is the case, we say that the iLa is reducible.

THEOREM 3. Let $(\mathfrak{G}, \sigma, Q)$ be a simple pseudo-riemannian iLa with canonical decomposition $\mathfrak{G}=\mathfrak{S}+\mathfrak{M}$. If $(\mathfrak{G}, \sigma)$ is reducible, then $\mathfrak{M}$ decomposes into the 
direct sum of two (ad $\mathfrak{H})$-invariant, irreducible, $Q$-isotropic, abelian subspaces $\mathfrak{N}_{1}$ and $\mathfrak{N}_{2}$, such that $\mathfrak{N}_{1}$ and $\mathfrak{N}_{2}$ are dual in $R^{1}$ under the pairing $\left(X_{1}, X_{2}\right)$ $\rightarrow Q\left(X_{1}, X_{2}\right), X_{1} \in \mathfrak{N}_{1}, X_{2} \in \mathfrak{N}_{2}$.

Proof. Decompose $\mathfrak{M}$ in to the direct sum of irreducible subspaces

$$
\mathfrak{M}=\mathfrak{A}_{1}+\mathfrak{A}_{2}+\cdots+\mathfrak{A}_{n} .
$$

This can be done by virtue of Lemma E. Let $\mathfrak{A}_{j}^{+}=\left\{X \in \mathfrak{M} \mid Q\left(X, \mathfrak{A}_{j}\right)=0\right\}$. Then $\mathfrak{A}_{j} \cap \mathfrak{U}_{j}^{\perp} \neq 0$, for otherwise the restriction of $Q$ on $\mathfrak{A}_{j}$ would be nondegenerate, contradicting Lemma $H$. It follows that $\mathfrak{A}_{j}^{\perp} \cap \mathfrak{A}_{j} \supset \mathfrak{A}_{j}$, i.e., $\mathfrak{A}_{j}$ is $Q$-isotropic for all $j$.

If $\mathfrak{U}_{1}+\mathfrak{A}_{2} \neq \mathfrak{M}$, then $\left(\mathfrak{A}_{1}+\mathfrak{U}_{2}\right)$ is not a $Q$-subspace and hence $\left(\mathfrak{U}_{1}+\mathfrak{U}_{2}\right)^{\perp}$ contains at least one of $\mathfrak{U}_{1}, \mathfrak{A}_{2}$. Thus $\mathfrak{A}_{1} \perp_{Q} \mathfrak{A}_{2}$ and consequently $\left(\mathfrak{A}_{1}+\mathfrak{U}_{2}\right)$ is $Q$-isotropic. Repeating this argument we find that the $Q$-isotropic subspaces $\mathfrak{A}_{1}, \mathfrak{A}_{2}, \cdots, \mathfrak{A}_{n-1}$ are pairwise orthogonal under $Q$. Therefore $\mathfrak{N}_{1}=\mathfrak{A}_{1}+\cdots+\mathfrak{A}_{n-1}$ is $Q$-isotrop ic. Put $\mathfrak{N}_{2}=\mathfrak{A}_{n}$; then $\mathfrak{N}_{2}$ is irreducible and $\mathfrak{M}=\mathfrak{N}_{1}+\mathfrak{N}_{2}$ (direct). Now, if $X_{1} \in \mathfrak{N}_{1}$ is such that $Q\left(X_{1}, \mathfrak{N}_{2}\right)=0$, then $Q\left(X_{1}, \mathfrak{M}\right)=0$ and hence $X_{1}=0$. Similarly, if $X_{2} \in \mathfrak{N}_{2}$ is such that $Q\left(\mathfrak{N}_{1}, X_{2}\right)=0$, then $X_{2}=0$; this proves the duality in $R^{1}$. The irreducibility of $\mathfrak{N}_{2}$ together with the fact that $Q\left(\left[X_{1}, H\right], X_{2}\right)$ $=Q\left(X_{1},\left[H, X_{2}\right]\right)$ shows that $\mathfrak{N}_{1}$, being dual to $\mathfrak{N}_{1}$, is itself irreducible. To complete the proof we need to show that $\mathfrak{N}_{1}$ and $\mathfrak{N}_{2}$ are abelian subalgebras. Let $X, Y$ be elements of $\mathfrak{N}_{1}$ and $Z, T$ be elements of $\mathfrak{M}$. Then $Q([[X, Y], Z], T)$ $=Q([[Z, T], X], Y)=0$, by virtue of Lemma $F$ and the fact that $\mathfrak{N}_{1}$ is invariant and $Q$-isotropic. Since $Z$ and $T$ are arbitrary, since $Q$ is nondegenerate and $\operatorname{ad}_{\mathfrak{m}} \mathfrak{H}$ is faithful, we conclude that $[X, Y]=0$, i.e., $\mathfrak{N}_{1}$ is abelian. The theorem now follows trivially.

We now specialize to the case where the pseudo-riemannian metric $Q$ is given by the Killing form $B$ of $(5$. Let $\theta$ be a $*$-automorphism of $\mathbb{5}$, commuting with $\sigma$, and let $\mathfrak{G}=\mathfrak{R}+\mathfrak{P}$ be the corresponding Cartan decomposition. Denote $\mathfrak{M} \cap \mathfrak{P}$ and $\mathfrak{M} \cap \Omega$ by $\mathfrak{M}_{p}$ and $\mathfrak{M}_{\mathfrak{\Omega}}$ respectively. Since $\theta(\mathfrak{M})=\mathfrak{M}$, we have $\mathfrak{M}=\mathfrak{M}_{\mathfrak{\Omega}}+\mathfrak{M}_{\mathfrak{p}}$. Let $\mathfrak{N}_{1}$ and $\mathfrak{N}_{2}$ be as in Theorem 3. Then $\theta\left(\mathfrak{N}_{1}\right)$ is an irreducible subspace of $\mathfrak{M}$, and hence either $\theta\left(\mathfrak{N}_{1}\right) \cap \mathfrak{N}_{1}=0$ or $\theta\left(\mathfrak{N}_{1}\right)=\mathfrak{N}_{1}$. If the latter case prevails, then $\mathfrak{N}_{1}=\mathfrak{N}_{1} \cap \mathfrak{R}+\mathfrak{N}_{1} \cap \mathfrak{P}$. Since $B$ is definite on $\mathfrak{R}$ and on $\mathfrak{P}, \mathfrak{N}_{1}$ cannot be $B$-isotropic, contradicting Theorem 3 . We therefore conclude that $\theta\left(\mathfrak{N}_{1}\right) \cap \mathfrak{N}_{1}=0$. It is now easy to see that we may choose $\mathfrak{N}_{2}=\theta\left(\mathfrak{N}_{1}\right)$ in Theorem 3 .

Choose a basis $e_{1}, e_{2}, \cdots, e_{m}$ for $\mathfrak{N}_{1}$; then $\theta\left(e_{1}\right), \theta\left(e_{2}\right), \cdots \theta\left(e_{m}\right)$ form a basis of $\mathfrak{N}_{2}$. Let $a_{i}=e_{i}+\theta\left(e_{i}\right)$ and $b_{i}=e_{i}-\theta\left(e_{i}\right), i=1,2, \cdots, m$. Then $a_{1}, a_{2}, \cdots, a_{m}$ form a basis of $\mathfrak{M}_{\mathfrak{R}}$, and $b_{1}, b_{2}, \cdots, b_{m}$ form a basis of $\mathfrak{M}_{p}$. We have, in particular,

$$
\operatorname{dim} \mathfrak{M}_{\boldsymbol{R}}=\operatorname{dim} \mathfrak{M}_{\mathfrak{p}} .
$$

THEOREM 4. Let $\mathfrak{G}=\mathfrak{H}+\mathfrak{M}$ and $\mathfrak{G}=\mathfrak{R}+\mathfrak{P}$ be the canonical and Cartan 
decompositions of a simple iLa $(\mathfrak{b}, \sigma)$. Then $(\mathfrak{5}, \sigma)$ is reducible if, and only if, there exists an element $e \in \mathfrak{H} \cap \mathfrak{P}$ whose centralizer in $\mathfrak{G}$ is $\mathfrak{h}$.

Proof. If $(\mathfrak{G}, \sigma)$ is reducible, then $\mathfrak{M}=\mathfrak{N}_{1}+\mathfrak{N}_{2}$ where $\mathfrak{N}_{2}=\theta\left(\mathfrak{N}_{1}\right)$ as in Theorem 3 and the remark immediately following it. A straightforward computation, using the facts that the restrictions of $B$ on $\mathfrak{M}$ and on $H$ are nondegenerate and that the representation $\mathfrak{H} \rightarrow \mathrm{ad}_{\mathfrak{M}} \mathfrak{H}$ is faithful, shows that the operator $D: \mathfrak{G} \rightarrow \mathfrak{G}$ defined by

$$
\begin{aligned}
& D(X)=0, \quad \text { if } X \in \mathfrak{H}, \\
& D(X)=X, \quad \text { if } X \in \mathfrak{N}_{1}, \\
& D(X)=-X, \text { if } X \in \mathfrak{N}_{2},
\end{aligned}
$$

is a derivation of $(5$ (i.e., $D[X, Y]=[D X, Y]+[X, D Y]$ ). Since every derivation of a simple Lie algebra $\mathfrak{G}$ is inner, we conclude that $D=$ ad $e^{\prime}$ for some $e^{\prime} \in \mathfrak{F}$. Let $e^{\prime \prime}=\left(e^{\prime}+\sigma e^{\prime}\right) / 2$. Then $e^{\prime \prime} \in \mathfrak{H}$ and ad $e^{\prime \prime}=D$. Finally, put $e=\left(e^{\prime \prime}-\theta e^{\prime \prime}\right) / 2$, where $\theta$ is the $*$-automorphism corresponding to $\mathfrak{G}=\mathfrak{K}+\mathfrak{P}$. One checks easily that $e$ satisfies the requirements.

Conversely, if $e \in \mathfrak{H} \cap \mathfrak{P}$ is given as stated, then the eigenvalues of $\operatorname{ad}_{\mathfrak{G}} e$ are all real. The eigenspaces decomposition

$$
\mathfrak{G}=\mathfrak{G}^{0}+\sum_{\lambda \in \Lambda} \mathfrak{F}^{\lambda},
$$

of $\left(\mathfrak{5}\right.$ with respect to ad $e$ has the following properties: (1) $\mathfrak{G}^{0}$ and $\mathfrak{G}^{\lambda}$ are $(\operatorname{ad} \mathfrak{H})-$ invariant for all $\lambda \in \Lambda$, where $\Lambda$ is the set of all non zero eigenvalues of $\operatorname{ad}_{\mathfrak{m}} e$; (2) $\mathfrak{G}^{\lambda} \perp_{B}\left(\mathfrak{G}^{\mu}\right.$ for $\lambda, \mu \in \Lambda, \mu+\lambda \neq 0$; and (3) each $\mathfrak{G}^{\lambda}$ is $B$-isotropic if $\lambda \neq 0$. Now Lemma $H$ implies that $\Lambda$ consists of two elements: $\Lambda=\{\lambda,-\lambda\}$. Thus

$$
\mathfrak{5}=\mathfrak{H}+\mathfrak{5}^{\lambda}+\mathfrak{W}^{-\lambda},
$$

since $\mathfrak{G}^{0}=\mathfrak{H}$. This obviously proves the theorem.

LeMMA I. Let $(\mathfrak{5}, \sigma)$ be a simple iLa with Cartan decomposition $\mathfrak{G}=\mathfrak{\Omega}+\mathfrak{P}$ and canonical decomposition $\mathfrak{G}=\mathfrak{H}+\mathfrak{M}$. Let the centrum of $\mathfrak{H}$ be denoted by $3(\mathfrak{H})$. Then

(1) $\mathfrak{Z}(\mathfrak{H}) \cap \mathfrak{P}$ (resp. $3(\mathfrak{H}) \cap \Re)$ is at most one-dimensional, and

(2) for each nonzero $e \in \mathfrak{Z}(\mathfrak{H}) \cap \mathfrak{P}($ resp. $t \in \mathfrak{Z}(\mathfrak{H}) \cap \mathfrak{R}), \operatorname{ad}_{\mathfrak{M}} e\left(\right.$ resp. $\left.\operatorname{ad}_{\mathfrak{M}} t\right)$ has exactly two eigenvalues $₫ a$ (resp. \pm ai), where a is a nonzero real number and $i=\sqrt{ }(-1)$.

Proof. We shall prove the second statement first. In proving Theorem 4, we have established the assertion concerning $e \in \mathfrak{Z}(\mathfrak{H}) \cap \mathfrak{P}$. We now consider an element $t \neq 0$ in $3(\mathfrak{H}) \cap \Re$. Obviously, all the nonzero eigenvalues of $\operatorname{ad}_{\mathfrak{G}} t$ are purely imaginary. Let $\mathfrak{G}_{c}$ be the complexification of $\mathfrak{G}$. Then $\mathfrak{G}_{c}$ is either simple or is 
the direct sum of two simple ideals. This together with Lemma $\mathrm{H}$ show that the eigenspaces decomposition of $\mathfrak{G}_{c}$ with respect to $\operatorname{ad}(t)$ consists of at most five pieces:

$$
\mathfrak{G}_{c}=\mathfrak{H}_{c}+\mathfrak{M}^{\lambda i}+\mathfrak{M}^{-\lambda i}+\mathfrak{M}^{\mu i}+\mathfrak{M}^{-\mu i} \quad(\lambda \neq \mu) .
$$

Put $\mathfrak{M}^{\lambda}=\mathfrak{M}^{\lambda i}+\mathfrak{M}^{-\lambda i}, \mathfrak{M}^{\mu}=\mathfrak{M}^{\mu i}+\mathfrak{M}^{-\mu i}$. Then $\mathfrak{M}^{\lambda} \perp_{B} \mathfrak{M}^{\mu}$ and $I^{\lambda}=\mathfrak{M}^{\lambda}+\left[\mathfrak{M}^{\lambda}, \mathfrak{M}^{\lambda}\right]$, $I^{\mu}=\mathfrak{M}^{\mu}+\left[\mathfrak{M}^{\mu}, \mathfrak{M}^{\mu}\right]$ are simple ideals of $\mathfrak{G}_{c}$ with $\mathfrak{G}_{c}=I^{\lambda}+I^{\mu}$.

Let $\theta$ be the $*$-automorphism corresponding to the given Cartan decomposition (extended to $\mathfrak{b}_{c}$ ). Since $t \in \mathfrak{R}$, we have

$$
\theta\left(\mathfrak{M}^{ \pm \lambda i}\right)=\mathfrak{M}^{ \pm \lambda i}, \theta\left(\mathfrak{M}^{ \pm \mu i}\right)=\mathfrak{M}^{ \pm \mu i} .
$$

Similarly, if $\tau$ denotes the conjugation in $\mathfrak{G}_{c}$ with respect to $\mathfrak{G}$, then

$$
\tau\left(\mathfrak{M}^{ \pm \lambda i}\right)=\mathfrak{M}^{\mp \lambda i},\left(\mathfrak{M}^{ \pm \mu i}\right)=\mathfrak{M}^{\mp \mu i} .
$$

Now decompose $t$ into $t^{\lambda}+t^{\mu}$ in accordance with the decomposition $\mathfrak{G}_{c}=I^{\lambda}+I^{\mu}$. $\operatorname{Then} \operatorname{ad}\left(t^{\lambda}\right)=\operatorname{ad}\left(t^{\lambda}\right) \mid I^{\lambda}$ and $\operatorname{ad}\left(t^{\mu}\right)=\operatorname{ad}(t) \mid I^{\mu}$. By virtue of formulae (4) and (5), 0 and $\tau$ preserves the decomposition $I^{\lambda}+I^{\mu}$, and consequently leave fixed the elements $t^{\lambda}$ and $t^{\mu}$, i.e., $t^{\lambda}$ and $t^{\mu}$ are in $\Re$. This shows that $\operatorname{ad}\left(t^{\lambda}\right)$ has eigenvalues 0 and $\pm i$ on $\mathfrak{M}_{c}$ hence on $\mathfrak{M}$; but this is impossible in view of the simplicity of $\mathfrak{G}$ and Lemma $\mathrm{H}$. Therefore either $\mathfrak{M}^{\lambda}=0$ or $\mathfrak{M}^{\mu}=0$. If follows that $\operatorname{ad}_{\mathfrak{M}} t$ has exactly two eigenvalues, and hence statement (2).

To prove the first assertion of the lemma, we let $e_{1}$ and $e_{2}$ be elements in $3(\mathfrak{H}) \cap \mathfrak{P}$. Multiplying these elements by a real constant if necessary, we may assume that $\operatorname{ad}_{\mathfrak{m}} e_{j}$ has eigenvalues $\pm 1(j=1,2)$. With respect to $\operatorname{ad}\left(e_{j}\right), \mathfrak{M}$ decomposes into the direct sum of eigenspaces:

$$
\mathfrak{M}=\mathfrak{M}_{j}^{1}+\mathfrak{M}_{j}^{-1} .
$$

Since $\operatorname{ad}\left(e_{1}\right)$ and $\operatorname{ad}\left(e_{2}\right)$ commutes and $\mathfrak{M}_{j}^{1}$ and $\mathfrak{M}_{j}^{-1}$ are irreducible, we conclude that either $\mathfrak{M}_{1}^{1}=\mathfrak{M}_{2}^{1}$ and $e_{1}=e_{2}$ or $\mathfrak{M}_{1}^{1}=\mathfrak{M}_{2}^{-1}$ and $e_{1}=-e_{2}$. In any case $\mathcal{Z}(\mathfrak{H}) \cap \mathfrak{P}$ is one-dimensional. Similarly, for elements $t_{1}$ and $t_{2}$ in $3(\mathfrak{H}) \cap \mathfrak{R}$, we may assume that $\operatorname{ad}_{\mathfrak{M}} t_{j}$ has eigenvalues $\pm i$. Decompose $\mathfrak{M}_{c}$ into the direct sum of eigenspaces:

$$
\mathfrak{M}_{c}=\mathfrak{M}_{j}^{i}+\mathfrak{M}_{j}^{-i}, \quad j=1,2 .
$$

We have either $\operatorname{ad}\left(t_{1}\right)=\operatorname{ad}\left( \pm t_{2}\right)$, in which case $t_{1}= \pm t_{2}$, or

$$
\mathfrak{M}_{c}=\mathfrak{M}^{i, i}+\mathfrak{M}^{i,-i}+\mathfrak{M}^{-i, i}+\mathfrak{M}^{-i,-i}
$$

(direct sum of eigenspaces). If the latter case prevail, the element $\left(t_{1}+t_{2}\right) / 2$ in $\mathfrak{R} \cap \mathcal{Z}(\mathfrak{H})$ has eigenvalues $0, i$, and $-i$ on $\mathfrak{M}_{c}$; this contradicts the second assertion of the lemma, which we have just established. Hence $\operatorname{dim}(3(\mathfrak{H}) \cap \Re) \leqq 1$.

The following theorem and its corollary were first proved by Borel and 
Lichnerowicz for the hermitian case, and were generalized, with essentially the same proof, by Berger to pseudo-hermitian iLa's (cf. [1]).

THEOREM 5. Let $(\mathfrak{G}, \sigma, Q)$ be a semi-simple pseudo-hermitian iLa. Let $\mathfrak{G}=\mathfrak{G}+\mathfrak{M}$ be the canonical decomposition of $(\mathfrak{5}, \sigma)$, and let $J$ denote the given complex structure on $\mathfrak{M}$. Then there exists an element $t \in \mathfrak{Z}(\mathfrak{M}) \cap \Re$ such that $J=\operatorname{ad}_{\mathfrak{m}} t$.

Proof. Consider arbitrary elements $X, Y, Z, T$ in $\mathfrak{M}$. Using Lemma $F$, we have

$$
\begin{aligned}
Q([[X, J Y], Z], T) & =Q([[Z, T], X], J Y)=-Q(J[[Z, T], X], Y) \\
& =-Q([[Z, T], J X], Y)=-Q([[J X, Y], Z], T) .
\end{aligned}
$$

It follows that $[X, J Y]+[J X, Y]=0$. Now, extend $J$ over $\mathfrak{G}$ by defining $J \mid \mathfrak{H}=0$. Then the extended $J$ is obviously a derivation and hence there exists $t \in \mathfrak{Z}(\mathfrak{H})$ such that $\operatorname{ad}_{\mathfrak{m}} t=J$. To see that $t$ is also in $\Re$, we observe that

$$
\mathfrak{Z}(\mathfrak{H})=\mathfrak{Z}(\mathfrak{H}) \cap \mathfrak{A}+\mathfrak{Z}(\mathfrak{H}) \cap \mathfrak{P}
$$

and $\exp (\operatorname{ad} t)$ is compact.

CoRollary A simple iLa $(\mathfrak{G}, \sigma)$ is pseudo-hermitian if, and only if, $3(\mathfrak{H}) \cap \Re$ is one-dimensional.

This is a direct consequence of the theorem and Lemma $\mathbf{I}$.

It is now obvious that, in a pseudo-hermitian iLa, the complex structure on $\mathfrak{M}$ is unique up to a sign.

THEOREM 6. In the canonical decomposition $\mathfrak{G}=\mathfrak{H}+\mathfrak{M}$ of a simple iLa $(\mathfrak{G}, \sigma)$, if the centrum $3(\mathfrak{H})$ of $\mathfrak{H}$ is two-dimensional, then $\mathfrak{5}$ admits a structure of complex Lie algebra, and with respect to this complex structure $\sigma$ is again an automorphism.

Proof. Let $\mathfrak{G}=\mathfrak{R}+\mathfrak{P}$ be a Cartan decomposition of $(\mathfrak{b}, \sigma)$. From the fact that $\mathfrak{Z}(\mathfrak{H})=\mathfrak{Z}(\mathfrak{Y}) \cap \mathfrak{R}+\mathcal{Z}(\mathfrak{Y}) \cap \mathfrak{P}$, we conclude that $\mathfrak{Y}(\mathfrak{H})$ is spanned by an element $e \in \mathfrak{P}$ and an element $t \in \mathfrak{R}$; furthermore, the eigenvalues of $\operatorname{ad}_{\mathfrak{m}} e$ can be assumed to be 1 and -1 , and that of $\operatorname{ad}_{\mathfrak{m}} t$ to be $i$ and $-i$. Let $\mathfrak{M}=\mathfrak{M}^{1}+\mathfrak{M}^{-1}$ be the eigenspaces decomposition of $\mathfrak{M}$ with respect to ad(e). We now define an operator $J: \mathfrak{G} \rightarrow \mathfrak{G}$ as follows. First we let

$$
J e=t, \quad J t=-e,
$$

and

$$
[e, J M]=[t, M] \text {, for all } M \in \mathfrak{M} \text {. }
$$

Since $\mathfrak{M}^{1}$ and $\mathfrak{M}^{-1}$ are abelian subalgebras and since $[\mathfrak{M}, \mathfrak{M}]=\mathfrak{H}$, we see that 
every $H \in \mathfrak{S}$ is of the form $\left[M_{1}, M_{-1}\right]=H$, where $M_{1} \in \mathfrak{M}^{1}$ and $M_{-1} \in \mathfrak{M}^{-1}$. We put

$$
J H=J\left[M_{1}, M_{-1}\right]=\left[J M_{1}, M_{-1}\right] .
$$

From (6) and (7), we deduce that

$$
\begin{aligned}
{[H, J M] } & =J[H, M], \text { for } H \in \mathfrak{H} \text { and } M \in \mathfrak{M} ; \\
J M & =\left\{\begin{array}{l}
\operatorname{ad}(t) M, \text { if } M \in \mathfrak{M}^{1}, \\
-\operatorname{ad}(t) M, \text { if } M \in \mathfrak{M}^{-1}
\end{array}\right.
\end{aligned}
$$

and finally, from (10),

$$
\left[M_{1}, J M_{-1}\right]=\left[J M_{1}, M_{-1}\right] \text {, for all } M_{1} \in \mathfrak{M}^{1}, M_{-1} \in \mathfrak{M}^{-1} .
$$

It is now a routine computation to show that

$$
\begin{aligned}
& J(J X)=-X, \text { for } X \in \mathfrak{G}, \text { and } \\
& {[J H, M]=J[H, M] \text {, for } M \in \mathfrak{M} .}
\end{aligned}
$$

This last formula, which is proved by considering first $M \in \mathfrak{M}^{1}$ and then $M \in \mathfrak{M}^{-1}$, together with (8) and (9), shows that

$$
[J X, Y]=[X, J Y]=J[X, Y],
$$

for all $X, Y$ in $\mathfrak{G}$. The theorem now follows.

A (real) iLa $(\mathfrak{b}, \sigma)$ is called pseudo-complex, if $\mathfrak{b}$ is a complex Lie algebra considered as a real Lie algebra of twice the complex dimension. If, furthermore, $\sigma$ is a complex authomorphism, then the $\mathrm{iLa}(\mathfrak{G}, \sigma)$ is said to be complex.

THEOREM 7. If an almost complex simple iLa $(\mathfrak{5}, \sigma)$ is reducible, then it is complex (indeed, $3(\mathfrak{H})$ is two-dimensional).

Proof. Let the almost complex structure be given by $J_{0}: \mathfrak{M} \rightarrow \mathfrak{M}$, and let $e \in \mathfrak{H}$, and $\mathfrak{M}=\mathfrak{M}^{1}+\mathfrak{M}^{-1}$ be as in the proof of Theorem 6. Since $J_{0}$ commutes with ad $(e)$, we have $J_{0}\left(\mathfrak{M}^{1}\right)=\mathfrak{M}^{1}$ and $J_{0}\left(\mathfrak{M}^{-1}\right)=\mathfrak{M}^{-1}$. Define $J: \mathfrak{M} \rightarrow \mathfrak{M}$ as follows:

and

$$
J\left|\mathfrak{M}^{1}=J_{0}\right| \mathfrak{M}^{1}, \quad J \mid \mathfrak{H}=0,
$$

$$
B\left(X_{1}, J X_{-1}\right)=B\left(J X_{1}, X_{-1}\right),
$$

for all $X_{1} \in \mathfrak{M}^{1}, X_{-1} \in \mathfrak{M}^{-1}$, where $B$ is the Killing form of $\mathfrak{G}$. Then, in virtue of the duality between $\mathfrak{M}^{1}$ and $\mathfrak{M}^{-1}$ under $B$ (see Theorem 3 ), $J$ is well defined. One verifies easily that $J \mid \mathfrak{M}$ defines a complex structure on $\mathfrak{M}$ and, using the nondegeneracy of $B$ on $\mathfrak{G}$, that $J$ is a derivation of $\mathfrak{b}$. It follows that there exists an element $t \in(\mathfrak{G}$ such that $\operatorname{ad}(t)=J$. Replacing $t$ by $(t+\sigma t) / 2$ if necessary, we 
may assume that $t \in \mathfrak{H}$. It is obvious that $\exp (\operatorname{ad} t)$ is compact, and hence $t \in \mathfrak{R}$. This proves that $\mathfrak{Z}(\mathfrak{H})$ is two-dimensional, and hence $(\mathfrak{G}, \sigma)$ is complex by Theorem 5.

THEOREM 8. Let $(\mathfrak{5}, \sigma)$ be a simple iLa which is not pseudo-complex. Then $(\mathfrak{G}, \sigma)$ is almost complex implies that it is pseudo-hermitian.

Proof. Let $\tilde{\mathfrak{G}}$ denote the underlying real Lie algebra of $\mathfrak{G}_{c}$, where $\mathfrak{G}_{c}$ is the complexification of $\mathfrak{G}$. Then $\sigma$ extends obviously over $\tilde{\mathfrak{G}}$, such that $(\tilde{\mathfrak{G}}, \sigma)$ is a pseudo-complex iLa. Notice that $(\mathfrak{G}, \sigma)$ is (simple and) not pseudo-complex means that $(\tilde{\mathfrak{G}}, \sigma)$ is simple. Let $\tilde{\mathfrak{G}}=\tilde{\mathfrak{H}}+\tilde{\mathfrak{M}}$ be the canonical decomposition of $(\tilde{\mathfrak{G}}, \sigma)$. A Cartan decomposition of $(\tilde{\mathfrak{G}}, \sigma)$ is given by $\tilde{\mathfrak{G}}=\mathfrak{U}+i \mathfrak{U}$, where $\mathfrak{U}=\mathfrak{R}+i \mathfrak{P}$ (cf. [2, p. 159]). Let the almost complex structure of $(\mathfrak{G}, \sigma)$ be given by $J: \mathfrak{M} \rightarrow \mathfrak{M}$. Then $\tilde{\mathfrak{M}}$ decomposes into $\tilde{\mathfrak{M}}=\tilde{\mathfrak{M}}_{1,0}+\tilde{\mathfrak{M}}_{0,1}$ with respect to $J$, where $J Z=i Z$ for $Z \in \mathfrak{M}_{1,0}$ and $J Z=-i Z$ for $Z \in \mathfrak{M}_{0,1}$. With respect to this decomposition, $(\tilde{\mathfrak{G}}, \sigma)$ is reducible. Therefore, by Theorem 4 , there exists an element $e^{\prime}$ in $3(\tilde{\mathfrak{H}}) \cap i \mathfrak{U}$ such that $\operatorname{ad}\left(e^{\prime}\right)=1$ on $\tilde{\mathfrak{M}}_{1,0}$ and -1 on $\tilde{\mathfrak{M}}_{0,1}$. Now, $\tilde{\mathfrak{H}} \cap i \mathfrak{U}=i(\mathfrak{R} \cap \mathfrak{H})+(\mathfrak{H} \cap \mathfrak{P})$, hence $e^{\prime}$ is of the form $i k+p(k \in \mathfrak{R}$ and $p \in \mathfrak{P})$. Recall that the elements in $\tilde{\mathfrak{M}}_{1,0}$ (resp. $\tilde{\mathfrak{M}}_{0,1}$ ) are of the form $X-i J X$ (resp. $X+i J X), X \in \mathfrak{M}$. Write the conditions $\left[e^{\prime}, Z\right]=Z$, if $Z \in \mathfrak{M}_{1,0}$, and $\left[e^{\prime}, Z\right]=-Z$, if $Z \in \mathfrak{M}_{0,1}$, in detail; we see that $p=0$ and $[k, X]=-J X$, for all $X \in \mathfrak{M}$. Thus $J=-\operatorname{ad}_{\mathfrak{m}} k, k \in \mathfrak{Z}(\mathfrak{S}) \cap \Omega$. The theorem now follows from the corollary of Theorem 5 .

As for the pseudo-complex iLa's, Berger has a complete description of them in $[1]$ (see p. 110).

\section{APPENDIX}

This appendix results from a conversation with J. A. Wolf.

Let $G$ be a Lie group with a finite number of connected components, and let $\Phi$ be a finite subgroup of the group of automorphisms of $G$. We shall show that there exists a maximal compact subgroup $K$ of $G$ which is stable under $\Phi$.

Consider the product space $L=\Phi \times G$, and identify $G$ with $\{1\} \times G$ in $L$, where 1 is the identity element in $\Phi$. We define a multiplication in $L$ as follows:

$$
(\alpha, g) \cdot\left(\beta, g^{\prime}\right)=\left(\alpha \beta, \beta^{-1}(g) \cdot g^{\prime}\right),
$$

for arbitrary elements $(\alpha, g)$ and $\left(\beta, g^{\prime}\right)$ in $L$. It is easy to verify that this multiplication, whose restriction on $G$ is the same as the group operation of $G$, turns $L$ into a Lie group. Now, $L$ has only a finite number of connected components the finite subgroup $\Phi \times\{1\}$ of $L$ is contained in a maximal compact subgroup $K_{1}$ of $L$ (cf. [6]); here 1 denotes the identity element of $G$. Since $G$ is a closed normal subgroup of $L$, the conjugacy theorem of maximal compact subgroups (ibid. Theorem 3.1) shows that $K=K_{1} \cap G$ is a maximal compact subgroup of $G$. In view of formula (13) and the fact that $\Phi \times\{1\} \subset K_{1}$, an element $(\alpha, k)$ of $L$ 
is in $K_{1}$ if, and only if, $(1, k)=k \in K$. We now claim that the maximal compact subgroup $K$ of $G$ is stable under $\Phi$. Indeed, for arbitrary $\alpha \in \Phi$ and $k \in K$, the element $(\alpha, k)$ is in $K_{1}$; hence $(\alpha, k) \cdot\left(\alpha^{-1}, 1\right)=(1, \alpha k) \in K_{1}$. But $(1, \alpha k)=\alpha k$ is also in $G$. Thus $\alpha k \in K_{1} \cap G=K$, which proves our assertion.

\section{BIBLIOGRAPHY}

1. M. Berger, Les éspace symétriques non-compacts, Ann. École Norm. Sup. (3) 74 (1957), 85-177.

2. S. Helgason, Differential geometry and symmetric spaces, Academic Press, New York, 1962.

3. S. Kobayashi and T. Nagano, On filtered Lie algebras and geometric structures. I, J. Math. Mech. 13 (1964), 875-908.

4. S. Kobayashi and K. Nomizu, Foundations of differential geometry, Vol. 1, Wiley, New York, 1963.

5. G. D. Mostow, On covariant fibering of Klein spaces, Amer. J. Math. 77 (1955), 105-112.

6. - Self-adjoint group, Ann. of Math. 62 (1955), 44-55.

7. É. J. Cartan, Groupes simples clos et ouverts et la géometrie riemanienne, J. Math. Pures Appl. 8 (1929), 1-33.

UNIVERSTTY OF CALIFornia,

Berkeley, California 